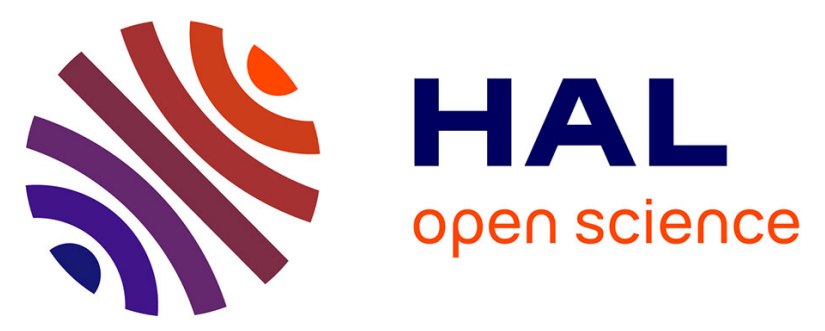

\title{
Conception de tableaux interactifs pour patients atteints de troubles sévères de la maladie d'Alzheimer (Tipatsma)
}

M Duchossoy, Christine Roosen, Nicolas Maranzana, C. Trivalle, Stéphanie Buisine

\section{To cite this version:}

M Duchossoy, Christine Roosen, Nicolas Maranzana, C. Trivalle, Stéphanie Buisine. Conception de tableaux interactifs pour patients atteints de troubles sévères de la maladie d'Alzheimer (Tipatsma): Design of interactive stations for patients suffering from severe impairmentscaused byAlzheimer'sdisease (the Tipatsma device). NPG: Neurologie - Psychiatrie - Gériatrie, 2015, 15 (89), pp.281-289. 10.1016/j.npg.2015.02.004 . hal-02423419

\section{HAL Id: hal-02423419 \\ https://hal.science/hal-02423419}

Submitted on 10 Jan 2020

HAL is a multi-disciplinary open access archive for the deposit and dissemination of scientific research documents, whether they are published or not. The documents may come from teaching and research institutions in France or abroad, or from public or private research centers.
L'archive ouverte pluridisciplinaire HAL, est destinée au dépôt et à la diffusion de documents scientifiques de niveau recherche, publiés ou non, émanant des établissements d'enseignement et de recherche français ou étrangers, des laboratoires publics ou privés. 


\title{
Conception de tableaux interactifs pour patients atteints de troubles sévères de la maladie d'Alzheimer (Tipatsma)
}

\section{Design of interactive stations for patients suffering from severe impairments caused by Alzheimer's disease (the Tipatsma device)}

\author{
M. Duchossoy ${ }^{a}$, C. Roosen ${ }^{b, *}$, N. Maranzana ${ }^{a}$, \\ C. Trivalle ${ }^{\mathrm{b}}$, S. Buisine ${ }^{\mathrm{a}, \mathrm{c}}$
}

a Arts et métiers ParisTech, LCPI, 151, boulevard de l'Hôpital, 75013 Paris, France

${ }^{b}$ Hôpital Paul-Brousse, AP-HP, 12, avenue Paul-Vaillant-Couturier, 94800 Villejuif, France

' Ei.Cesi, Irise, 93, boulevard de la Seine, BP 602, 92006 Nanterre cedex, France

\section{MOTS-CLÉS}

Maladie d'Alzheimer ; Troubles de la déambulation ;

Thérapie non médicamenteuse ; Protocole d'évaluation ; Tableau interactif

\begin{abstract}
Résumé Les troubles de la déambulation et de la manipulation incessante d'objets sont un phénomène fréquent chez les malades à un stade avancé de la maladie d'Alzheimer. Leur prise en charge est très complexe pour les institutions et peu de solutions satisfaisantes ont jusqu'à présent été proposées. Tableau interactif pour patients atteints de troubles sévères de la maladie d'Alzheimer (Tipatsma) se veut porteur d'une solution à destination de ces patients et de leur entourage. S'inscrivant dans une proposition thérapeutique non médicamenteuse, il met à portée de main un support d'activités sensorimotrices et cognitives placé sur le parcours de déambulation. Cet article retrace le processus itératif de conception et d'évaluation de la matérialisation de ce dispositif, dans une démarche constructiviste. Les deux itérations successives ont permis de mieux cerner les besoins des patients et d'apporter de nouvelles pistes pour l'amélioration des solutions proposées.
\end{abstract}

* Auteur correspondant.

Adresse e-mail : christine.roosen@pbr.aphp.fr (C. Roosen). 


\section{KEYWORDS}

Alzheimer's disease; Perambulation; Non-pharmacological treatment; Evaluation protocol; Interactive station
Summary Perambulation (wandering) and manipulative restlessness are very common in advanced Alzheimer's disease. Management of these behavioral disorders is very complex for institutions, and few satisfactory solutions have been put forward. The Tipatsma device (an interactive station for patients suffering from severe impairments caused by Alzheimer's disease) provides a solution for these patients and their families. As part of a non-pharmacological treatment, when positioned on the perambulation route, it offers a medium for sensory-motor and cognitive activities. In this paper, we report on the iterative design of this device and its evaluation, in a constructivist approach. The two successive iterations provided insight into patients' needs and new opportunities to improve this device.
Au début de ce projet, en 2006, la maladie d'Alzheimer touchait 26,6 millions de personnes dans le monde et ce chiffre risque de quadrupler d'ici 2050 [1-3]. C'est la forme la plus fréquente de démence et la première cause de dépendance lourde chez la personne âgée. Les comportements dans la maladie d'Alzheimer dépendent notamment de la localisation de l'atteinte neurologique, de l'évolution des symptômes, de l'état psychologique et physique du patient, de son parcours de vie et de son environnement. Cependant, les troubles du comportement sont un motif fréquent de consultation aux urgences et d'entrée en institution [4-6]. Une des difficultés concerne les patients déambulants à un stade avancé de la maladie [7]. La prise en soin de ces patients hyperactifs, par ailleurs lourdement handicapés pour gérer tous les actes de leur vie quotidienne, constitue un enjeu complexe car elle nécessite une surveillance accrue et contraignante engendrant beaucoup d'anxiété et de problèmes organisationnels au sein des services concernés. N'ayant pas de moyens et de dispositifs adaptés aux comportements de ces patients, le personnel soignant, souvent démuni face aux crises d'hyperactivité, se voit parfois obligé de recourir à une prescription médicamenteuse [8], voire à une contention physique. Ces solutions, difficilement vécues par le patient, sa famille et le personnel, ne peuvent être envisagées à long terme. De plus, les psychotropes sont peu efficaces pour lutter contre les troubles comportementaux et engendrent des risques supplémentaires pour les patients, des effets indésirables, voire une hausse de mortalité [9].

Diverses formes d'approches thérapeutiques non médicamenteuses (psychologiques, sociales, stimulantes, rééducatives...) sont de plus en plus utilisées pour accompagner les patients en s'adaptant à leurs capacités. Elles ont pour objectif d'améliorer le quotidien du patient et de son entourage, englobant aussi bien des questions de diagnostic, de soin que d'accompagnement, de qualité de vie et de préservation de la dignité [10]. Les activités thérapeutiques basées sur le ludique, l'expression artistique, le social, les ateliers réminiscence, sportifs, etc. sont proposées dès que possible dans les divers lieux d'accueil et de soins. Ayant pour but de soutenir les personnes tant sur le plan psychologique que cognitif, elles sont malheureusement souvent abandonnées alors que les troubles se majorent. Les difficultés d'attention, de concentration, d'expression, de compréhension font souvent perdre pied et confiance et ainsi au stade sévère de la maladie, on assiste à un repli ou à une agitation qui rendent difficile la participation des patients aux activités en groupe, voire même en situation individuelle. Ce repli prend souvent la forme de l'apathie, du sommeil mais aussi de l'entrée petit à petit dans un monde apparemment éloigné de l'environnement tangible.

Le projet Tableau interactif pour patients atteints de troubles sévères de la maladie d'Alzheimer (Tipatsma) vise à outiller la prise en charge non médicamenteuse des troubles sévères de la maladie d'Alzheimer ou apparentées, en particulier pour les patients déambulants qui interviennent avec force sur leur environnement et qui ne relèvent plus, ou que de façon très furtive, d'ateliers d'expression et de stimulation cognitive classiques. On peut dire qu'il met à disposition une intervention sur-mesure, pour répondre au besoin, désir, de chaque patient en fonction des comportements particuliers qu'il développe et du moment où il peut les mener. Faisant écho sur certains plans aux principes sous-jacents de la pédagogie active tels ceux énoncés, par exemple, par Maria Montessori, il pourrait aussi s'apparenter au processus describe, investigate, create, and evaluate (DICE) décrit plus récemment par Fraker et al. [9].

\section{Le projet Tipatsma}

Ce projet a été initié au sein d'unités spécialisées dans le soin et l'accompagnement de personnes atteintes de maladies de types Alzheimer et apparentées (SSR Alzheimer, hôpital Paul-Brousse, AP-HP). De nombreuses années de travail clinique et d'observation de ces patients ont montré qu'en dehors des crises aiguës et soudaines signant le plus souvent l'apparition de douleurs physiques ou psychiques, les troubles du comportement telles la déambulation, l'hyperactivité et la manipulation d'objets survenant alors que la maladie évolue, dévoilent des capacités physiques et cognitives toujours présentes et qui peuvent s'organiser. Même s'ils ne respectent pas tous les enchaînements attendus, ces comportements, perçus comme étranges, voire perturbateurs, qui, au fil des jours vont souvent se ritualiser sont, semble-t-il, pour les patients un moyen de lutter contre leurs angoisses et pour rester en lien avec leur être et leur histoire de vie personnelle et sociale. Ainsi, au travers de propos recueillis, on les verra «aller chercher les enfants à l'école», «faire les lits, ranger les chambres», «préparer les repas », « aplanir ou nettoyer le sol », « réparer le chauffage», «aider à déménager», «travailler», etc. 


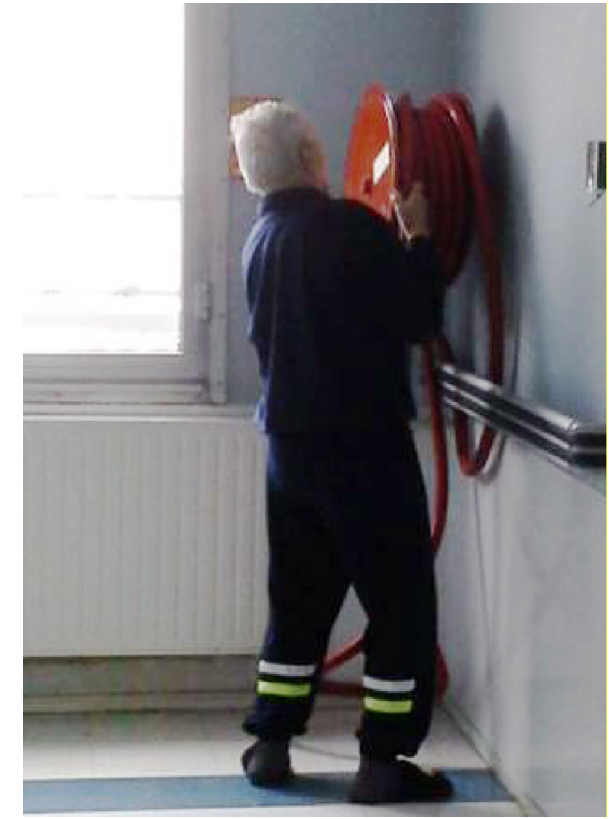

Figure 1. Patient manipulant des équipements de sécurité dans le couloir de l'hôpital.

Il semble important d'encourager ces comportements porteurs de stratégies et témoignant d'activités cognitives et d'apprentissage.

Cependant, ces activités sont menées avec ténacité et souvent en force dans un environnement qui ne leur est pas adapté. Elles amènent les patients à manipuler certains objets de façon inappropriée (par exemple, des équipements de sécurité, Fig. 1), à risquer de se blesser ou encore à détériorer leur lieu de vie (par exemple, dérégler ou démonter un radiateur). Les aidants ou le personnel soignant, qui doivent parfois intervenir en urgence, au risque de susciter une situation conflictuelle et d'agressivité en retour, ont donc tendance à décourager, voire empêcher ces activités.

Tipatsma est un concept breveté [11] qui vise à résoudre ce paradoxe en offrant aux patients des supports adaptés à leur besoin de manipulation afin de soutenir leur désir d'être actifs et de trouver à leur manière des solutions. Il s'agit de «tableaux interactifs» robustes constitués de matériaux nobles et d'éléments attractifs, placés sur le parcours de déambulation, qui sont destinés à être des supports d'activités sensorimotrices et cognitives. L'enjeu est de reconnaître l'importance des actions physiques, permettre une activité libre et autonome vers des objets et matériaux non dangereux, stimuler les sens, les fonctions cognitives, maintenir les capacités présentes, créer un plaisir immédiat porteur d'un retour de l'estime de soi et diminuer le stress lié aux situations d'agressivité.

\section{Processus de conception}

Pour matérialiser le concept de Tipatsma, nous avons dû relever un défi de conception. En effet, compte tenu des particularités des utilisateurs, la plupart des méthodes d'analyse du besoin et de conception centrées utilisateurs [12] se retrouvaient inopérantes. En effet, même pour les soignants et pour les proches des patients, il est très complexe à ce stade de la maladie d'accéder aux processus mentaux et à la subjectivité des patients, d'interpréter leurs comportements et leur discours: ils ne peuvent donc généralement pas exprimer leur besoin, donner leur avis, ni se soumettre à un protocole de test utilisateur.

En conséquence, notre stratégie a consisté à inscrire le projet dans une approche itérative et constructiviste [13], en définissant de façon conjointe le besoin et la solution tout au long du projet. Ce type d'approche a, par ailleurs, été décrit plus récemment par Liem en 2014 [14]. Concrètement, nous avons décidé de réaliser un premier prototype en nous appuyant sur un cahier des charges fonctionnel issu uniquement de l'expérience clinique, puis d'introduire le prototype dans le lieu de vie des patients afin d'obtenir un retour d'expérience et d'affiner le cahier des charges fonctionnel. Ce dernier a enfin été pris en compte pour concevoir deux nouveaux prototypes.

\section{Première génération de Tipatsma}

La matérialisation d'un premier prototype s'est basée sur les spécifications suivantes:

- stimuler les sens: la vue, le toucher, l'ouie et l'odorat (le goût a été exclu pour des raisons d'hygiène et de faisabilité en environnement collectif);

- stimuler la motricité: soutenir des activités d'exploration, des actions à réaliser porteuses d'effets ;

- préserver la dignité et la sécurité des patients: le design du produit et les matériaux utilisés doivent inscrire le produit dans un univers non infantilisant mais néanmoins robustes et non dangereux.

Le prototype $[11,15]$ prend la forme d'un tableau mural (Fig. 2). L'utilisation de plusieurs matériaux (bois rouge d'eucalyptus, aluminium, PVC, ABS, plexiglas) offre des contrastes visuels, des textures variées à explorer par le toucher et stimule l'odorat (principalement par l'odeur du bois et de l'huile de lin dont il est imprégné). Pour combiner ces matériaux sans compromettre la solidité de l'ensemble, nous les avons organisés en couches et avons usiné des cavités dans cet assemblage. Une manivelle de machineoutil est intégrée au tableau pour attirer les comportements de manipulation: lorsqu'elle est actionnée, elle entraîne l'allumage de LED vertes qui diffusent leur lumière dans toute la couche de plexiglas. Un bouton poussoir positionné à l'intérieur d'une cavité déclenche un petit ventilateur qui exhale l'odeur du bois et de l'huile de lin et allume une lumière bleue. Une autre cavité est équipée de cordes de cithare qui résonnent sous les doigts. Enfin, de petits coussins en textile sont fixés dans certaines cavités par des aimants et peuvent donc être arrachés, emportés ou replacés dans leur logement. Une fois fabriqué, ce prototype a été installé en octobre 2010 au sein de l'unité Victor-Hugo de l'hôpital Paul-Brousse à Villejuif.

\section{Évaluation du premier prototype}

La littérature comporte un manque considérable au niveau de l'évaluation des produits pour les patients atteints de 


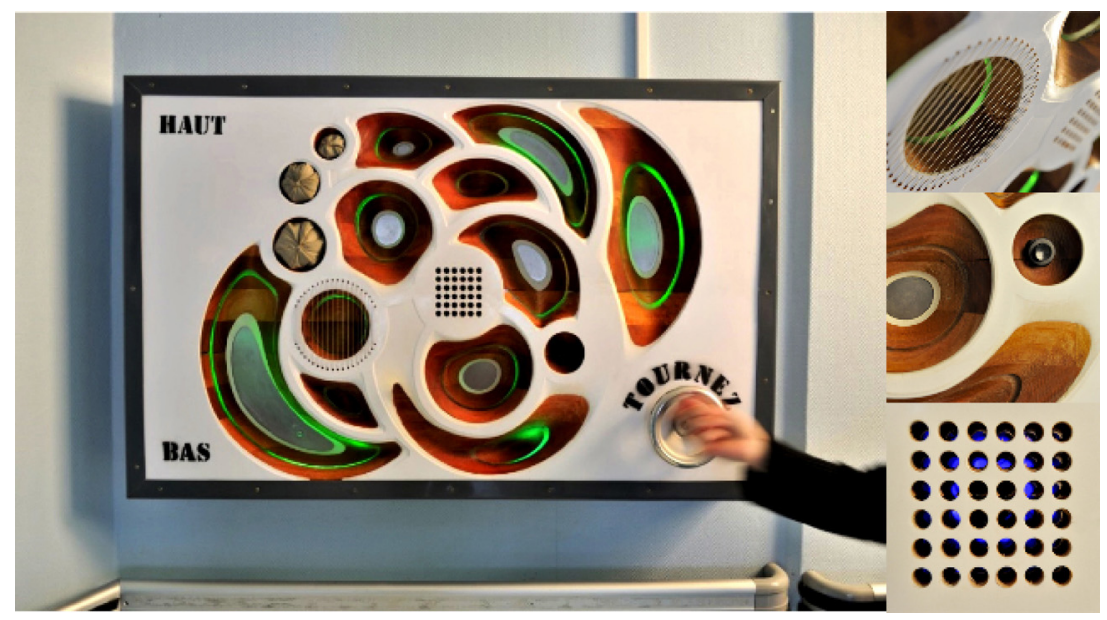

Figure 2. Le premier prototype Tipatsma.

la maladie d'Alzheimer, et en particulier, à ce stade de la maladie. Les études portant sur les démarches thérapeutiques évaluent davantage des activités que des produits. L'évaluation dans un tel contexte comporte trois difficultés majeures: les problèmes neuropsychologiques sévères rencontrés par les patients [16], le manque de précédents dans la littérature [17] et le manque de produits spécifiques sur le marché.

Compte tenu des troubles causés par la maladie d'Alzheimer [18], aucun des principaux outils d'évaluation de l'utilisabilité [19] ne peut être appliqué dans ce contexte $[20,21]$. Ainsi, l'agitation du patient, l'instabilité de ses émotions, ses grandes difficultés d'expression, ses troubles de la mémoire pour répondre à des consignes constituent de véritables obstacles pour permettre une évaluation. Seules les observations qui ne nécessitent pas de conditions particulières de tests ni de participation active du patient sont donc envisageables.

L'évaluation clinique des voies non médicamenteuses pour les personnes atteintes de la maladie d'Alzheimer se confronte également à plusieurs obstacles [22]: l'effectif restreint des échantillons, les difficultés d'interprétations des résultats du fait de l'hétérogénéité des populations (types de démences et sévérité des troubles), l'absence de comparabilité des études (contextes et protocoles d'évaluation) et la multiplicité des critères d'évaluation. L'utilisation d'outils reconnus et standardisés peut s'avérer être une piste intéressante allant dans ce sens [23].

Nous avons donc conçu un protocole d'évaluation pluriel ayant pour but d'analyser l'utilisabilité du dispositif pour les patients, son acceptabilité pour les soignants et les aidants, et son intégration dans le service.

\section{Protocole d'évaluation}

Pour évaluer l'utilisabilité du premier prototype, nous avons conçu un nouveau modèle d'inspection expérimentale se situant au croisement des méthodes d'évaluation ergonomique et de l'évaluation clinique. L'utilisabilité est une notion fondamentale en ergonomie, elle se définit comme le degré selon lequel un produit peut être utilisé, par des utilisateurs identifiés, pour atteindre des buts définis avec efficacité, efficience et satisfaction, dans un contexte d'utilisation spécifié $[12,24]$. Aux critères d'efficacité, d'efficience et de satisfaction, certains auteurs ajoutent des critères comme l'apprentissage et l'attrait du dispositif [25].

Pour estimer l'utilisabilité du prototype selon ces critères, nous avons combiné des observations auprès de patients et des entretiens et questionnaires auprès des aidants et des soignants. L'échantillon de patients a été constitué de la manière suivante. Parmi les 27 patients de l'unité, nous avons sélectionné 12 d'entre eux présentant des troubles sévères de la maladie d'Alzheimer $(M M S<10)$. Nous avons estimé leur profil comportemental à l'aide du Cohen Mansfield agitation inventory (CMAI) [26] et du neuropsychiatric inventory (NPI) [27], et les avons ensuite répartis en deux groupes: le groupe cible et le groupe secondaire. Le groupe cible était constitué de trois patients (deux hommes et une femme de plus de 80 ans) correspondant à notre utilisateur principal, le patient déambulant hyperactif (CMAl $\geq 150 / 205$ et $\mathrm{NPI} \geq 100$ ). Le groupe secondaire était constitué des neuf autres patients (quatre hommes et cinq femmes de plus de 80 ans) aux capacités physiques et cognitives similaires, mais non-déambulants. L'accord préalable d'un membre de la famille a été obtenu pour tous les patients, ainsi que l'accord des soignants.

Nous avons observé le comportement spontané du groupe cible, sans intervention de notre part (Fig. 3). Les sessions d'observation ont été menées in-situ par demi-journées, deux fois par semaine sur une période de neuf semaines (en tout, environ 60 heures d'observation), tantôt en matinée, tantôt en après-midi pour couvrir différentes plages horaires et ainsi avoir plus de chances d'observer des variations comportementales. La Fig. 4 présente la grille d'observation conçue pour cette étude. Des méthodes d'évaluation clinique nous ont permis de compléter nos observations, par exemple avec une évaluation de l'agitation [28]. Nous n'avons pas utilisé d'observation vidéo, cette solution ayant été écartée par le service pour des raisons éthiques et médicales.

En ce qui concerne le groupe secondaire, nous avons accompagné les patients un à un devant le dispositif pour répondre à des consignes très simples (exemple: «appuyez sur le bouton»; «voyez-vous la lumière verte?»). Nous 


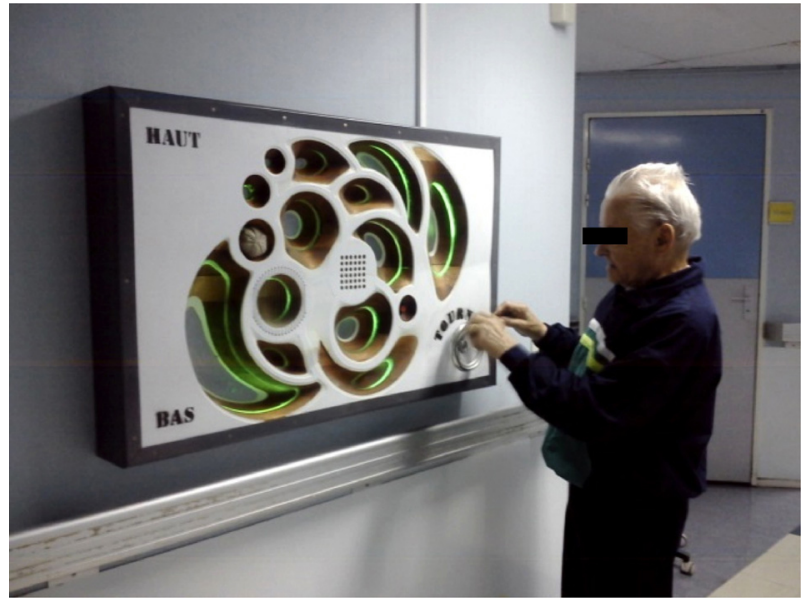

Figure 3. Un patient du groupe cible manipulant le prototype.

avons recueilli leurs verbalisations et leurs attitudes de manière similaire au groupe cible.

Pour compléter ces données, nous avons interrogé 19 soignants (infirmiers, aides-soignants, médecins, psychomotricien et psychologue) sur leurs propres observations de l'utilisation du prototype par les patients, sur les retombées par rapport à leur prise en charge et à l'organisation du service. Enfin, 4 aidants (issus de 4 familles différentes, de différentes générations et avec différents liens de parenté vis-à-vis des patients) ont aussi été sollicités pour évaluer l'acceptabilité du dispositif d'un point de vue éthique et recueillir leurs avis de manière générale.

\section{Résultats}

Le taux de passage des patients déambulants devant le dispositif est de l'ordre d'un passage toutes les trois minutes, mais le taux d'observation du dispositif est de l'ordre de $11 \%$. Parmi les patients ayant regardé le dispositif lors de leur passage, $60 \%$ ont marqué un arrêt ou un ralentissement, ce qui indique que le dispositif, une fois remarqué, retient l'attention.

La manivelle est l'activité la plus attractive et la plus appréciée des patients. Elle représente $40 \%$ des manipulations des patients déambulants et est manipulée en premier par les patients non déambulants dans $80 \%$ des cas. C'est également l'activité la plus pertinente du point de vue du personnel soignant. La cithare vient en seconde position avec $35 \%$ des manipulations des patients déambulants, puis l'exploration libre des cavités (15\% des manipulations). La cithare est attractive, mais elle produit peu de réponses stimulantes à la manipulation: les patients ont des difficultés à produire des sons car les cordes doivent être grattées du bout des doigts, voire avec les ongles, en un geste qui requiert de la dextérité. Enfin, les petits coussins amovibles, qui avaient été volontairement placés en hauteur pour ne pas être trop rapidement accessibles, se sont avérés positionnés trop haut, compte tenu de la diminution de taille des patients avec l'âge et de certaines pathologies articulatoires.

La satisfaction des patients a été estimée à l'aide de leurs expressions faciales, de leurs verbalisations spontanées et de la durée de manipulation des activités. La manivelle suscite souvent des expressions de surprise ou de contentement au moment de la découverte de l'effet lumineux. En outre, nous avons choisi un modèle de manivelle de machine-outil, ce qui a amené certains patients à évoquer des souvenirs de leurs études ou de leur passé professionnel. Les verbalisations spontanées des patients concernaient également les formes et l'esthétique du tableau: plusieurs patients se sont montrés intrigués, ont qualifié les formes «d'étranges» ou de «peu communes». Deux hommes ont perçu dans le tableau une évocation de l'univers automobile, d'un tableau de bord, une femme a fait référence à des instruments musicaux et une autre à un paysage et à la nature.

En ce qui concerne l'acceptabilité, nous avons constaté que le projet était très favorablement jugé par les soignants et les aidants. Par exemple, à la question «Trouvezvous le concept Tipatsma intéressant?», 21 personnes sur 23 ont répondu «tout à fait». Dans les commentaires libres, les personnes interrogées ont exprimé beaucoup d'enthousiasme, ont souligné l'intérêt de proposer des activités aux patients, d'améliorer leur cadre de vie, ou encore d'entretenir leurs capacités préservées. Pour les soignants, l'intérêt du dispositif réside surtout dans l'activité apportée aux patients, plus que dans le plaisir ou l'amusement. Les points mentionnés par les soignants comme étant les plus importants concernent à la fois la sécurité (exemple: positionnement des activités pour ne pas créer de déséquilibre ou de risque de chute chez les patients), les fonctionnalités du prototype (exemple: proposer plusieurs activités motrices simples comme des mouvements latéraux et verticaux en plus de la rotation) ainsi que son esthétique (exemple : intégrer plus de couleurs et d'éléments lumineux pour attirer le regard).

\section{Seconde génération de Tipatsma}

À partir de l'ensemble des évaluations recueillies, nous avons été en mesure de formuler de nouvelles spécifications pour concevoir une seconde génération de prototypes:

- attirer l'attention: le premier prototype n'attirant pas suffisamment le regard des patients, nous avons décidé d'introduire des couleurs lumineuses, assez vives et des contrastes plus marqués. Les nouveaux modèles sont aussi de plus grande taille, avec des contrastes sur les proportions des éléments afin d'en dynamiser l'aspect visuel. Enfin, pour faire ressortir les activités lorsque le patient passe à côté du tableau, nous avons décidé de diminuer l'épaisseur du support et de créer des activités en relief plutôt qu'en creux;

- soutenir des activités variées: le design abstrait du premier prototype a permis aux patients d'y projeter des centres d'intérêt différents, mais il a aussi suscité de la perplexité et parfois dérouté l'action. Pour les nouveaux modèles, nous avons choisi des formes plus communicantes (linéarité pour l'un, circularité pour l'autre) pour mieux orienter l'action des patients. Nous avons conservé le principe de la manivelle qui a eu beaucoup de succès et ajouté d'autres mouvements rotatifs, horizontaux et verticaux. Nous avons aussi multiplié les petits éléments 


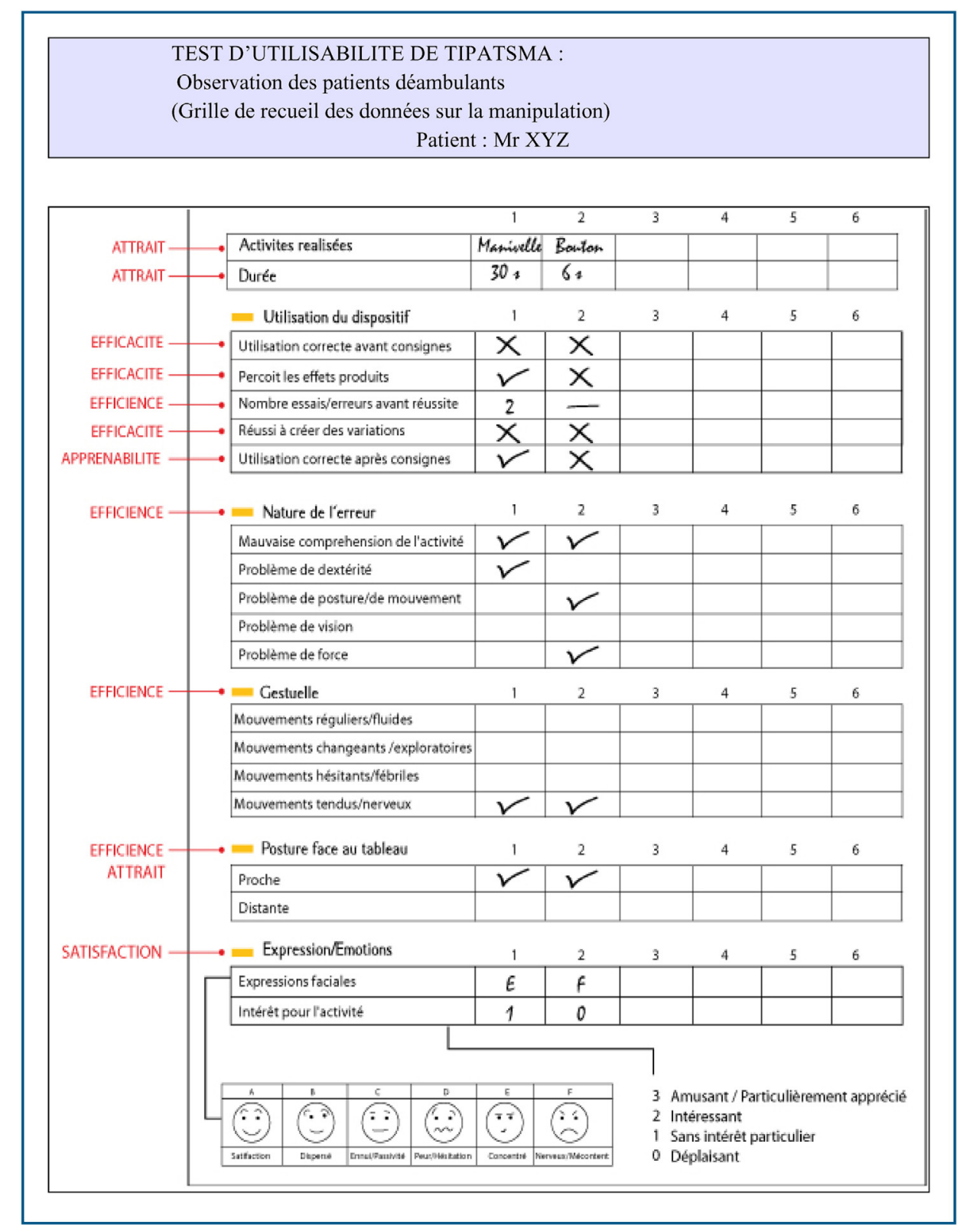

Figure 4. Grille d'évaluation de l'utilisabilité.

permettant de varier les matières, les textures et les types de manipulation;

- soutenir l'évocation du passé, la réminiscence: le succès de la manivelle est aussi dû au fait qu'il s'agisse d'un objet du «passé». Nous avons introduit dans les nouveaux modèles divers objets du passé, par exemple un cadran de téléphone rotatif, des pampilles de rideaux, etc ;

- conserver une esthétique chaleureuse, qualitative et robuste: les matériaux du premier prototype ont été appréciés, en particulier le bois. Pour la fabrication des nouveaux prototypes, nous avons essentiellement utilisé du bois et du métal ;

- améliorer l'accessibilité des activités : pour ne pas obliger le patient à manipuler des éléments en hauteur, les nouveaux tableaux ont été étirés dans le sens de la longueur.
Pour une bonne accessibilité, la hauteur des activités doit être comprise entre 90 et $160 \mathrm{~cm}$.

La seconde génération de Tipatsma comprend deux prototypes. Le premier (Fig. 5) est une composition de formes circulaires. Un cadran d'horloge abrite un jeu d'engrenages qui peuvent être mis en mouvement par une manivelle. Quatre pastilles lumineuses sont disposées sur la surface du tableau: elles peuvent être commandées par trois boutons poussoirs et un potentiomètre pour contrôler les couleurs et leur rythme de variation. Plusieurs accroche-torchons sont également intégrés au tableau, dans lesquels on peut trouver des carrés de textile amovibles de différentes couleurs, épaisseurs, douceurs - à emporter, à replacer. D'autres éléments peuvent être explorés: des boules de caoutchouc à presser, un panneau aimanté, un petit émetteur radio, etc. 


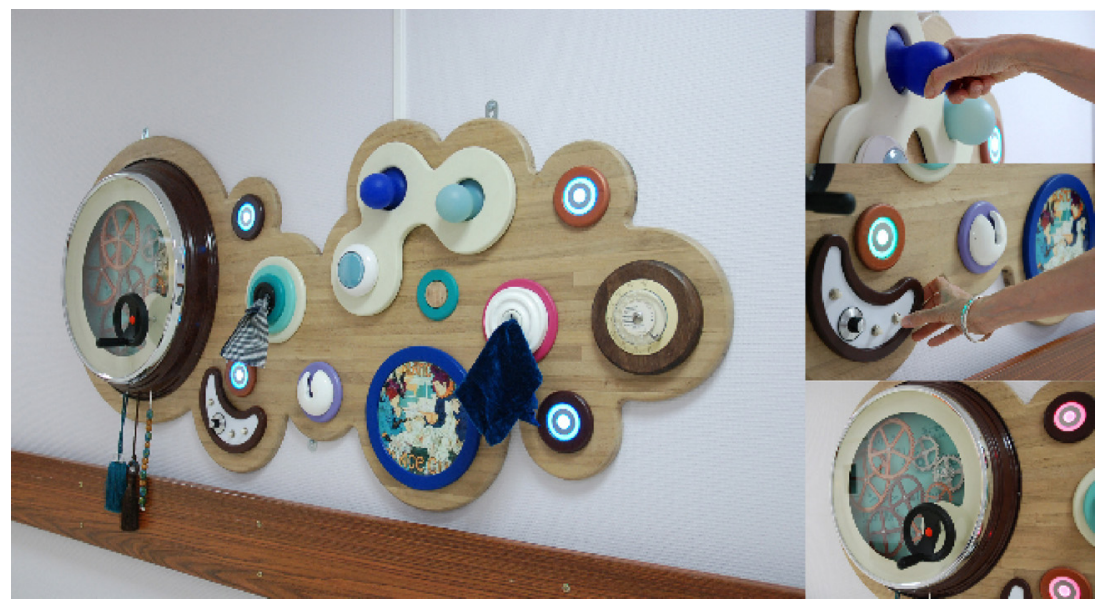

Figure 5. Le prototype circulaire de la seconde génération de Tipatsma.

Enfin, un cadran de téléphone rotatif rappelle une manipulation d'autrefois, associée au bruit caractéristique du disque qui se remet en place après composition d'un numéro. Ce tableau qui mêle à la fois une esthétique industrielle, mécanique et domestique, mise sur la diversité.

Le second prototype, plus épuré, est composé de formes linéaires (Fig. 6). L'activité principale est un circuit de manipulation sous forme de rails dans lesquels circulent des boutons de porte. Le rail principal est en métal et une branche du circuit est en plexiglas. Dans cette dernière, lorsque le bouton arrive en butée, il éclaire un ruban lumineux qui parcourt toute la branche. Enfin, un carillon mobile est aussi intégré à ce tableau de sorte à proposer une activité musicale nécessitant moins de dextérité que la cithare de la première génération de Tipatsma.

\section{Discussion}

Les prototypes de seconde génération de Tipatsma sont installés et opérationnels à l'hôpital Paul-Brousse de Villejuif depuis février 2012.

Au-delà de la conception et de la réalisation des Tipatsma, un point important à ne pas négliger et qui est difficile à définir est le positionnement des tableaux dans les espaces de déambulation. Cette notion est présente depuis les prémisses du projet Tipatsma. Nous y avons attaché une attention toute particulière en prenant en compte les données environnementales ainsi que celles de plusieurs années d'observation in situ des patients déambulants. Nous sommes dans la situation paradoxale suivante: le tableau doit être à la fois visible, repérable et en même temps placé en un lieu protégé des regards et de l'agitation pour favoriser l'attention, la concentration et préserver le sentiment de liberté et d'indépendance du patient. Il est également souhaitable que le patient puisse visualiser le produit de face lors de sa déambulation. Or en pratique, il est souvent difficile de trouver un tel positionnement dans les espaces de déambulation: les bouts de couloirs sont généralement occupés et éclairés par des fenêtres ou des portes-fenêtres. Son positionnement est alors à préconiser à une intersection de couloirs ou en fond de couloir sur des parois libres que l'on découvre en ralentissant la marche et en amorçant un demi-tour. Dans le positionnement en intersection, cela permet certes d'augmenter le taux de passage devant le dispositif, y compris des non-déambulants, mais en fond de couloir, cela privilégie la tranquillité nécessaire pour que le patient puisse soutenir son attention et mener son activité manipulatoire à l'abri des regards et des commentaires perturbateurs.

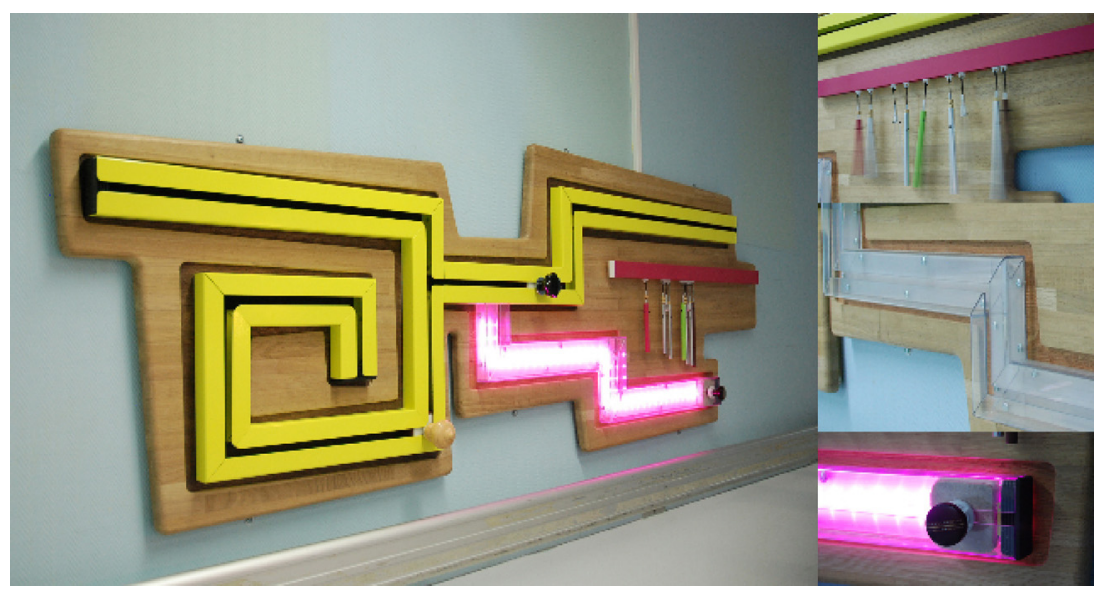

Figure 6. Prototype linéaire de la seconde génération de Tipatsma. 
Les échanges observés entre les patients non déambulants et les enfants des visiteurs familiaux autour des tableaux nous ont conduits à repenser et diversifier le positionnement de ceux-ci dans l'espace de déambulation. Tout en gardant prioritairement des emplacements dans des zones à l'abri de l'agitation et des regards, l'idée serait d'en disposer également non loin de celles à plus grande circulation pour permettre à tous d'en faire usage: patients, soignants, aidants, visiteurs.

Un autre point important de cette discussion concerne l'évaluation des Tipatsma et de leur impact. La poursuite logique de cette étude serait d'en faire une évaluation formelle, d'un point de vue ergonomique comme le prototype de première génération, mais aussi d'un point de vue clinique. Pour évaluer les bénéfices apportés par ces dispositifs dans le cadre de la prise en charge des troubles sévères de la maladie d'Alzheimer, il faudrait mobiliser plusieurs services dans plusieurs hôpitaux de sorte à toucher un nombre suffisant de patients cibles - environ une vingtaine [29], voire le double si on choisit d'avoir un groupe témoin. Il faudrait ensuite caractériser à l'aide d'échelles standardisées les troubles cognitifs, affectifs et comportementaux de l'ensemble des patients avant l'introduction des Tipatsma. L'étape suivante serait de recueillir des observations du comportement des patients, avec ou sans Tipatsma, pendant une durée de 8 à 12 semaines consécutives. Concernant ces observations et évaluations du comportement des patients, il est à noter que : contrairement à la plupart des activités thérapeutiques, les dispositifs de Tipatsma sont installés en permanence sur les murs de l'espace de vie et s'utilisent en toute autonomie et liberté par les patients. Il n'est donc pas nécessaire de mettre en place des sessions ponctuelles, animées par les membres de l'équipe soignante pour procéder à l'évaluation. Cela offre plus de souplesse et de flexibilité pour l'observation mais introduit d'autres contraintes.

Pour une meilleure fiabilité des observations, il nous a été parfois recommandé de recourir à la vidéo et de confier l'annotation comportementale à plusieurs experts en insu. Cependant, l'introduction de caméras même discrètes dans un lieu de vie (et non un laboratoire ou salle dédiée) pose problème surtout vis-à-vis d'une population présentant fréquemment des interprétations délirantes et des hallucinations et mérite d'être discutée sur le plan éthique et médical avant leur utilisation. Enfin, à l'issue de cette période, il faudrait à nouveau évaluer les troubles cognitifs, affectifs et comportementaux de l'ensemble des patients pour constater, ou pas, un bénéfice apporté par les Tipatsma.

Pour réaliser cette évaluation clinique, il faudrait disposer de plusieurs exemplaires de chaque prototype, permettant de généraliser l'approche (services avec des organisations, des architectures différentes) et de toucher un échantillon de patients suffisant et stable. Il faudrait pour cela amorcer l'industrialisation des Tipatsma pour bénéficier de moyens de production et de fabrication professionnels car nos tableaux sont pour le moment des exemplaires uniques.

\section{Conclusion}

Tandis que la littérature actuelle fait état des bénéfices des activités thérapeutiques non médicamenteuses
$[30,31]$, cette étude nous a apporté des informations plurielles et complémentaires qui ont trait à d'autres notions importantes: l'utilisabilité d'un produit spécifique avec ses critères d'efficacité, d'efficience, d'attrait, d'apprentissage et de satisfaction, son acceptabilité et ses impacts au sein de l'environnement médical. Comme attendu, Tipatsma répond à un réel besoin des patients (manipuler pour préserver leurs capacités, voire pour lutter contre la maladie), mais aussi des soignants et des aidants (offrir aux patients les moyens d'exercer ce besoin sans se mettre en danger ou détériorer leur lieu de vie). Cette étude nous a appris qu'il n'est, cependant, pas si simple de matérialiser ces solutions: amener les patients jusqu'aux dispositifs ne peut se faire qu'en jouant sur des facteurs sensoriels, perceptifs et attentionnels. Il faut aussi leur proposer des activités variées, qui répondent au maximum d'envies individuelles, et concevoir des dispositifs robustes, sécurisés et respectueux des patients. Cette étude nous a aussi permis d'identifier de nombreuses pistes pour poursuivre la conception de nouveaux Tipatsma, par exemple, l'utilisation d'objets du passé pour susciter la réminiscence et le plaisir, la déclinaison en gamme ou en éléments modulaires.

Trois prototypes sont désormais installés dans deux unités spécialisées du pôle gériatrie de l'hôpital PaulBrousse. Quoique informelle, l'observation quotidienne de leur utilisation in situ permet de conforter l'intérêt qu'ils représentent [32]. Tipatsma constitue un réel potentiel du point de vue des professionnels de terrain, des aidants familiaux et des distributeurs et acheteurs. Le projet a aussi été récompensé par plusieurs prix, montrant l'intérêt des acteurs socioéconomiques et institutionnels pour le concept (prix de recherche et d'innovation Charles-Foix en 2010, «Coup de cœur» des trophées du Grand Âge et du Bien Vieillir en 2013 et prix de «l'innovation technique au service des patients » des Trophées APinnov 2013). De plus, le peu de solutions existantes pour endiguer ces comportements difficiles à gérer nous incite à poursuivre notre démarche vers l'industrialisation.

Comme acteur d'une thérapie non médicamenteuse, notamment lors de troubles du comportement dits «perturbateurs », Tipatsma a sa place dans les unités spécialisées des centres hospitaliers, Ehpad, maisons de retraites, centres d'accueil de jour, domiciles, voire les MAS et autres établissements spécialisés. Au-delà des personnes concernées en premier lieu par ce dispositif, Tipatsma a déjà montré qu'il permettait de susciter des échanges intergénérationnels et laisse entrevoir également une extension de son utilisation par des porteurs d'autres handicaps.

\section{Remerciements}

Sont chaleureusement remerciés: la direction de l'hôpital Paul-Brousse et le personnel soignant et médical des unités SSR Alzheimer de son pôle gériatrie, notamment le Dr F. Tourres; les accompagnants de la première heure de ce projet, B. Zimmer d'Aresa/Sol'iage, L. Dechesne du CFI, ainsi que l'OTT\&PI de l'AP-HP; HandiTecAM et France Alzheimer 93 qui ont financé la première génération de Tipatsma; le pôle allongement de la vie Charles-Foix et OSEO région île-de-France qui ont financé la seconde 
génération de Tipatsma; E. Inacio et F. Lesage pour leur inestimable contribution.

\section{Déclaration d'intérêts}

Les auteurs déclarent ne pas avoir de conflit d'intérêt potentiel en rapport avec cette publication. Stéphanie Buisine, Nicolas Maranzana et Christine Roosen sont co-inventeurs du brevet «tableau de manipulation pour patients atteints de la maladie d'Alzheimer» no FR 2010/0055754.

\section{Références}

[1] Ferri CP, Prince M, Brayne C, et al. Global prevalence of dementia: a Delphi consensus study. Lancet 2006;366:2112-7.

[2] Brookmeyer R, Johnson E, Ziegler-Graham K, et al. Forecasting the global burden of Alzheimer's disease. Alzheimer's Dementia 2007;3:186-91.

[3] United Nations, department of economic and social affairs, population division (2007). World population prospects: the 2006 revision, highlights. Working paper No. ESA/P/WP.202.

[4] Nourhashémi F, Andrieu S, Sastres N, et al. Descriptive analysis of emergency hospital admissions of patients with Alzheimer disese. Alzheimer Dis Assoc Disord 2001;15:21-5.

[5] Yaffe K, Fox P, Newcomer R, et al. Patients and caregiver characteristics and nursing home placement in patients with dementia. JAMA 2002;287:2090-7.

[6] Luppa M, Luck T, Brähler E, et al. Prediction of institutionalisation in dementia. A systematic review. Dement Geriatr Cogn Disord 2008;26:65-78.

[7] Rolland Y, Gillette-Guyonnet S, Nourhashémi F, et al. Déambulation et maladie de type Alzheimer. Étude descriptive. Programme de recherche Real.Fr sur la maladie d'Alzheimer et les filières de soins. Rev Med Interne 2003;24:333-8.

[8] Herrmann N, Lanctôt KL. Pharmacologic management of neuropsychiatric symptoms of Alzheimer disease. Can J Psychiatry 2007;52:630-46.

[9] Fraker J, Kales HC, Blazek M, et al. The role of the occupational therapist in the management of neuropsychiatric symptoms of dementia in clinical settings. Occup Ther Health Care 2014;28:4-20.

[10] Pancrazi MP, Métais P. Prise en charge non médicamenteuse dans les démences sévères. Psychol Neuropsychiatr Vieil 2005;3(Suppl. 1):S42-50.

[11] Roosen C, Inacio E, Buisine S, et al. Tableau de manipulation pour patients atteints de la maladie d'Alzheimer. Brevet français FR 2010/0055754 et international WO 2012/007484. http://www.google.com.tr/patents/WO2012007484A1?cl=fr\& $\mathrm{hl}=\mathrm{fr}$

[12] ISO 9241-210:2010. Ergonomics of human-system interaction - Part 210: human-centered design for interactive systems. Genève: International Organization for Standardization. http://www.iso.org/iso/home/store/catalogue_ics/catalogue detail_ics.htm?csnumber $=52075$

[13] Visser W. La conception: de la résolution de problèmes à la construction de représentations. Travail Hum 2009;72:61-78.

[14] Liem A. Toward prospective reasoning in design: an essay on relationships among designers' reasoning, business strategies, and innovation. Le Travail Humain 2014;77:91-102.
[15] Inacio E. Tipatsma : proposition non médicamenteuse à destination de patients atteints de troubles sévères de la maladie d'Alzheimer ou apparentées. Mémoire de master recherche innovation conception ingénierie, arts et métiers ParisTech, 2010.

[16] David R, Mulin E, Leone E, et al. Les symptômes psychologiques et comportementaux dans la maladie d'Alzheimer. Neurologie.com 2010;2:171-5. http://www.jle.com/e-docs/00/04/ 60/7F/vers_alt/VersionPDF.pdf

[17] Marshall MJ, Hutchinson SA. A critique of research on the use of activities with persons with Alzheimer's disease: a systematic literature review. J Adv Nurs 2001;35:488-96.

[18] Lacomblez L, Mahieux-Laurent F. Les démences du sujet âgé. Paris: Pathologie-Science, éditions John Libbey Eurotext; 2003.

[19] Nielsen J. Usability engineering. Boston: Academic Press; 1993.

[20] Duchossoy $M$. Conception et matérialisation d'une gamme de produits sensorimoteurs et cognitifs pour patients en stade avancé de la maladie d'Alzheimer et élaboration d'un protocole d'évaluation spécifique. Mémoire de master recherche innovation conception ingénierie, arts et métiers ParisTech, 2011.

[21] Duchossoy M, Buisine S, Maranzana N, et al. Conception d'un protocole d'évaluation ergonomique d'un produit destiné aux patients atteints de troubles sévères de la maladie d'Alzheimer, Confère $2011,18^{\mathrm{e}}$ colloque sur les sciences de la conception et de l'innovation.

[22] Agence nationale d'accréditation et d'évaluation en santé (Anaes), service évaluation technologique. Prise en charge non médicamenteuse de la maladie d'Alzheimer et des troubles apparentés, mai 2003.

[23] Dorenlot $P$. Démence et interventions non médicamenteuses: revue critique, bilan et perspectives. Psychol Neuropsychiatr Vieil 2006;4:135-44.

[24] Shackel B. Usability - context, framework, design and evaluation. In: Shackel, Richardson, editors. Human factors for informatics usability. Cambridge: Cambridge University Press; 1991. p. 21-38.

[25] Brangier E, Barcenilla J. Concevoir un produit facile à utiliser: adapter les technologies à l'homme. Paris: Éditions d'Organisation; 2003.

[26] Micas M, Ousset PJ, Vellas B. Évaluation des troubles du comportement. Présentation de l'échelle de Cohen Mansfield. Rev Fr Psychiatr Psychol Med 1997;7:151-7.

[27] Cummings JL, Mega M, Gray K, et al. The neuropsychiatric inventory: comprehensive assessment of psychopathology in dementia. Neurology 1994;44:2308-14.

[28] Burns A, Lawlor B, Craig S. Rating scales in old age psychiatry. Br J Psychiatry 2002;180:161-7.

[29] Seux ML, de Rotrou J, Rigaud AS. Les traitements de la maladie d'Alzheimer. Psychiatr Sci Hum Neurosci 2008;6:82-90.

[30] Wang KL, Hermann C. Pilot study to test the effectiveness of healing touch on agitation in people with dementia. Geriatr Nurs 2006;27:34-40.

[31] Amieva H, Dartigues JF. ETNA3, a clinical randomized study assessing three cognitive-oriented therapies in dementia: rationale and general design. Rev Neurol 2013;169:752-6.

[32] Roosen C, Duchossoy M, Inacio E, et al. Tableau interactif pour patients atteints de troubles sévères de la maladie d'Alzheimer: du travail clinique de terrain à la conception et réalisation d'une solution industrialisable. Congrès Européen de Stimulation Cognitive, 2014. 